\title{
Assessing the influence of restoration works on an artificialized river morphology
}

\author{
J. Botella ${ }^{1}$, Y. Brizard ${ }^{2}$, D. Chaisemartin ${ }^{1} \&$ R. Nicolau ${ }^{1}$ \\ ${ }^{I}$ Research Group on Water Soil and Environment, \\ University of Limoges, France \\ ${ }^{2}$ Vienne Basin Planning Association, France
}

\begin{abstract}
The Glane River is a French stream highly artificialized. It displays 20 transversal structures inducing rupture in the continuity. In the context of the Water Framework Directive, several restoration works have been scheduled between 2014 and 2016. A station in the head of the watershed including 3 successive weirs intended for removal has been selected for evaluating the river response to incoming changes. A specific methodology has been applied in order to assess the morphological evolution of the stream. It includes regular measurements of transversal and longitudinal geometric key values, and granulometric composition. The serial of measurements made before the first removal have helped to highlight reductions of sediment and biological communities continuity across the linear implied in the study. Particular degradation among diversity of hydraulic profiles and size classes of substrate have been identified in areas between weirs. The gradual decrease of the hydrological dynamics and the proportion of lotic fish species from downstream to upstream were observed.

The first weir removal was conducted in July 2014, inducing direct release of large amounts of fines from the reservoir. Campaigns have been made between 3 and 5 months after the beginning of works upstream and downstream of the removed structure. A gradual deposition of fine sediments has been observed downstream while their withdrawal from the reservoir resulted in the emergence of coarser material. The most significant observation of these mid-term measurements is the channelization of the upstream linear in parallel with the reduction of the bed width and augmentation of lotic profiles encountered.

Keywords: weir removal, morphology, sediment transport, dynamic profiles, habitats, stream response.
\end{abstract}




\section{Introduction}

Small dams found in streams are considered as an ecological issue by their local impact on natural environment. Although of modest size, they induce similar impacts to those of bigger ones, accentuated by their presence in great number over linear. Impacts generated by these structures on aquatic environments involve several fields of study such as status of biological communities, nutrient dynamics, or morphology $[1,2]$. In this article, work is focused on sedimentary, river morphology, habitats aspects and their relationship.

A stream is a complex environment, based on a delicate balance between all its parameters. Modification of one of them will influence the evolution of the whole system. Evolution of the stream sedimentary behaviour is one of the key points of this balance. So, one of the main impacts generated by the presence of a transversal obstacle is the physical barrier of sediment transfer along the river. Upstream of the weir a global phenomenon of sediment deposition is observed due to the decrease of water velocity [3]. Downstream of the structure, deficiency in new material and increase of velocity induced erosion with a channel incision [4], until equilibrium or non-erosive structure was reached.

The imposition of lentic profiles upstream of the structure [5] leads to a lack of riffles and lotic portions in the area of influence. This lack of flowing profiles diversity induced degradation of habitat quality [6].

Concerning old structures, an artificial balance was established in the river and it is important to consider any stream restoration operation as a disturbance and a reconfiguration of an environment at equilibrium [7].

Morphological changes induced by removal operation of a transversal structure take place during the first year after works [8]. Observations made during and after operations are generally transitions of the upstream pond to lotic systems with a quick transport of accumulated sediments upstream to downstream [4]. The drying of lateral areas where vegetation rapidly grows, and decrease of bed width upstream of the removed weir are also observed [9]. While the characteristics of the transport dynamics within the system are specific to each steam, there is generally a reduction in fine sediments in the upstream area, discharged on a linear more or less extended downstream [3].

Few studies deal with the removal of small transversal structures, and most of them are focused on operations performed on large rivers and high dams. Thus, the temporal and geographical scales of disturbances related to the presence or removal of small dam are still poorly known. These small structures are mainly considered as a local disturbance with poor impact on the whole river. Nevertheless, presence of numerous and close weirs as observed in many rivers, generate a greater impact and global disturbance of the stream easily perceptible [6].

The purpose of this work was to follow the removal operation of a weir located in the head of watershed of a highly artificialized French stream: the Glane River. The work is focused on the evolution of morphology and habitat characteristics of the upstream and downstream linear at both short and medium term. A methodology used by the French water national office (ONEMA) has been 
adapted to our needs and applied to reflect in the most accurate way the geometry of the river. We also mainly based our analysis on the work of Malavoi and Souchon [10] concerning grain-size and habitat parameters.

\section{Methods and material}

\subsection{Study area}

The Glane River is located in the Haute-Vienne area in the center of France. The river displays 26 transversal obstacles, resulting in a global stepping rate of $16.2 \%$ over its $43 \mathrm{~km}$. Restoration operations have been programmed on 16 of these structures between 2013 and 2016.

The study area (Fig. 1) has been defined at the head of the watershed .It includes three weirs displayed consecutively on a 1 kilometer long linear. In July 2014, the middle weir was removed. The previous weir is the Aval Rabaud, ranging from 0.9 to 1.3 meters in height. It underwent the complete deletion of the artificial part, until the bedrock natural obstacle. The reservoir upstream of the structure benefited during the works phase of the filling of a lateral annex (Fig. 2).

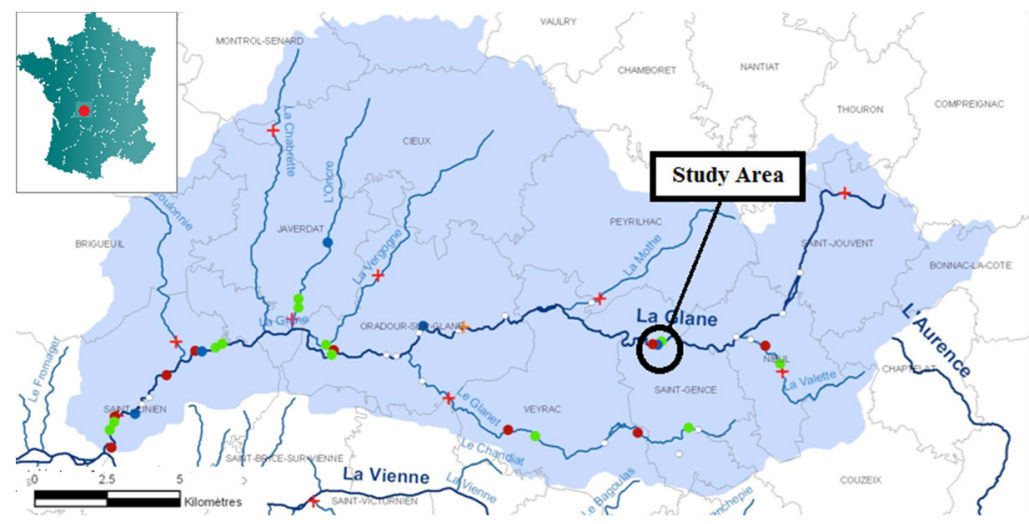

Figure 1: $\quad$ Study area. Each dot represents a transversal structure.
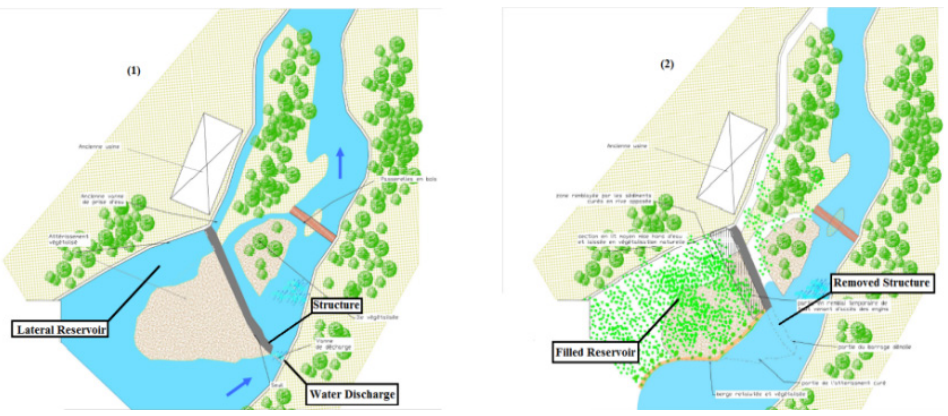

Figure 2: Planned removal of Aval Rabaud weir. 


\subsection{Methodology and analyses}

On the study site, 4 different areas have been defined:

- Zone 1 downstream of the group of structures ;

- Zone 2 between the downstream weir and Aval Rabaud;

- Zone 3 between Aval Rabaud and the upstream weir ;

- Zone 4 upstream of the group of structures.

Longitudinal profile was also made by the measurement of bed altitude along the river. Measurements have been performed every 10 meters using an infrared leveller (Fig. 3).

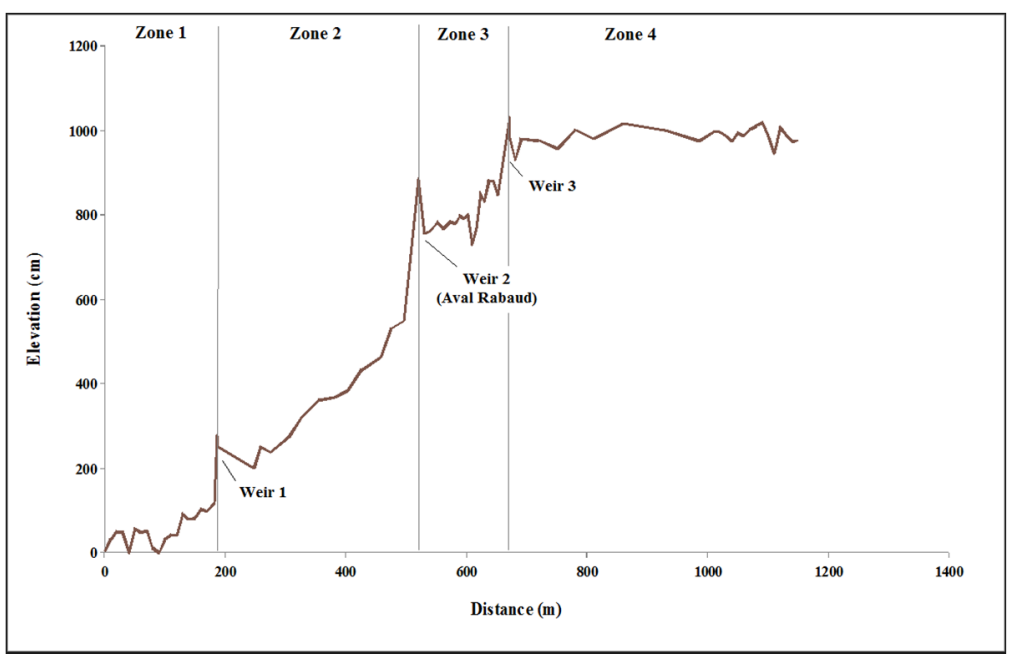

Figure 3: Longitudinal profile of study area.

The monitoring of solid particles transport has been performed upstream and downstream using:

- $\quad$ turbidity measures realised by autonomous probes (Exowater, EXO2)

- $\quad$ and TSS realised by filtration through $0.45 \mathrm{~mm}$ nitrocellulosic membrane. Water samples were obtaining using automatic samplers (Sigma, 900p).

Two measurement campaigns have been realised before and after removal work. Frequency of measurement has been fixed to 1 hour.

Along the river, transversals transects has been defined every 10 meters. For each of them, it has been noted:

- A description of banks (materials, nature of the habitats, thickness and type of side vegetation and presence of lateral erosion);

- $\quad$ Immerged bed width and full bed width (limit of overflowing);

- $\quad$ Flow profiles encountered (rapid, riffle, shallow lotic, shallow lentic, deep lentic);

- Local grain size and water depth every 1/10 of immerged bed width. Two grain sizes were described, one for dominant and one for coarser substrate. 
Application of this methodology for a 6 meters full bed width river over a 150 meters linear took half a day for 3 persons.

Habitats were assessed by the particle size noticed in the bed substrate. In this work, a grid based on the works of Malavoi and Souchon [10], and Frissel et al. [11] was used to distinguish 6 different habitats.

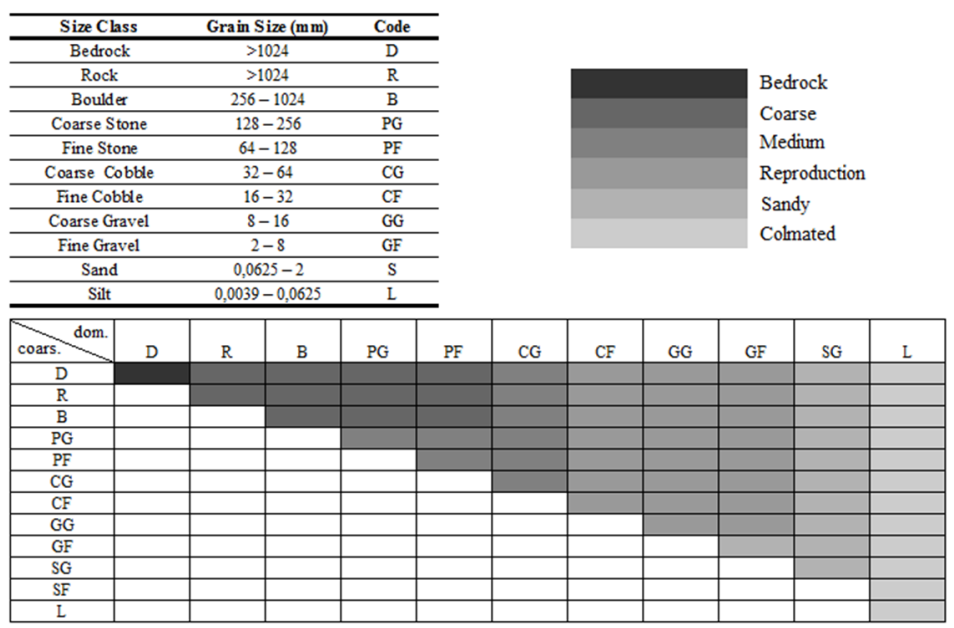

Figure 4: Grain size exploitation and habitats grids.

For the initial state all the fourth zones have been characterised.

After the removal of "Aval Rabaud" weir, only zones $2(330 \mathrm{~m})$ and $3(150 \mathrm{~m})$ have been described. Zones 1 and 4 have been protected from the work by the presence of upstream and downstream wears.

Pre-removal campaigns have been conducted between 10 and 4 months before the beginning of works and post-removal campaigns between 3 and 6 months after.

\section{Results and discussion}

Measurement campaigns performed before the weir removal have shown a highly impacted environment. Longitudinal profile highlighted a strong fragmentation of study site as evidenced in Fig. 3 by slope breach. Modification of natural slopes is also noticeable, with a flatten slope upstream weirs. The presence of sedimentfilled reservoir directly above the weirs can explain the slope decrease.

Regards to flow profile (Fig. 5), gradual augmentation of lentic profiles throughout upstream progression can be observed. Impact of weirs 2 (between zones 2 and 3 ) and 3 (between zones 3 and 4) were clearly noticed on upstream flow profiles. In fact, an increase of lentic profiles can be noticed between zones 2 and $3(10 \%)$ and between zones 3 and $4(50 \%)$. The influence of weirs seems stronger in zone 4 with a configuration favouring stagnation and sediment retention ( $86.7 \%$ of lentic flow). However, weir 1 (between zones 1 and 2) seems to have lower effect on flow profiles. Rapid and riffle decrease in favour of shallow 
lotic profile and deep lentic profile appears but fraction of lentic profiles keeps close to $20 \%$. This slight influence can be attributed to the advanced degradation state of the weir.

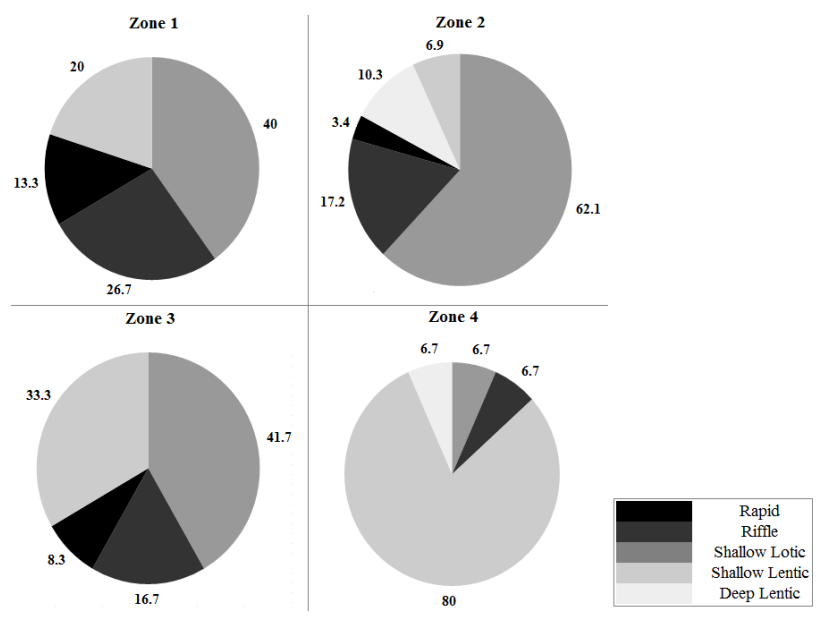

Figure 5: Flow profiles before removal.

Habitat study shows the consequence on sediment transport of the increase of lentic profile observed below (Fig. 6). Downstream to upstream, the increase of fine substrate (sandy and colmated) less favourable to life can be noticed: zone 1: 36.1\%; zone 2: 32.4; zone 3: 71.1, and zone 4: $95.8 \%$. One more time, impact of weir 1 is clearly less problematic than the 2 others. In comparison to zone 3 and 4 , zone 1 and 2 downstream of Aval Rabaud, present a greater diversity in available habitats. All habitats are present at percentage located between 10 and $30 \%$.

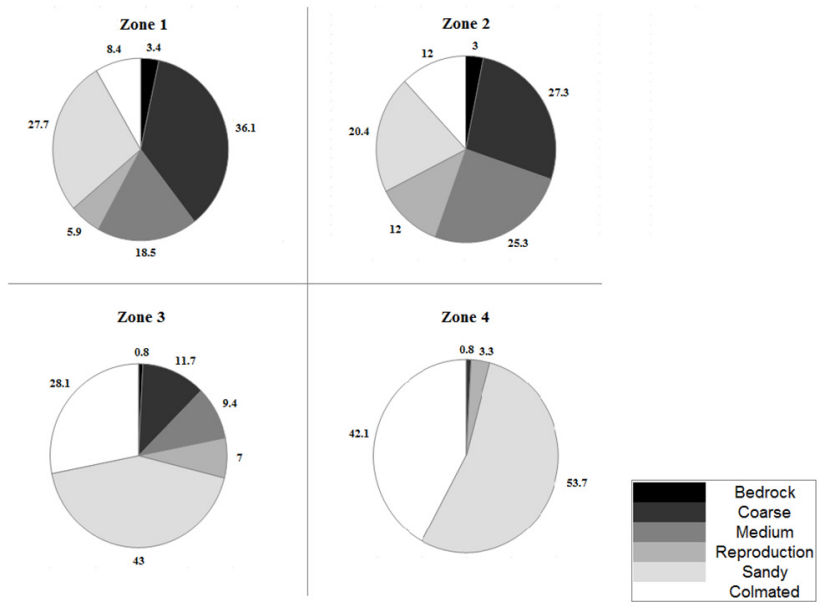

Figure 6: Habitats before removal. 
Fish communities inventory has been conducted by the Haute-Vienne fishermen association during October 2013. Inventory has not been conducted in zone 3, due to the reduced linear $(150 \mathrm{~m})$ in this sector not enabling completion of the measures. However the field surveys combined with previous observations leave few doubts that fish communities are under-represented on this particular zone. Structure of communities pointed out the strong fragmentation of the ecosystem. Zone 2 (constrained area of $330 \mathrm{~m}$ between weirs 1 and 2), despite presenting diversity of habitats and flow profiles (Figs 5 and 6), contains a weak biomass $\left(302 \mathrm{~g} / \mathrm{m}^{2}\right)$. Contrary to the previous, zones 1 and 4 show high biomass (up to $1500 \mathrm{~g} / \mathrm{m}^{2}$ ). These differences can be attributed to the part of river disconnected: zone 1: $7.5 \mathrm{~km}$; zone $2: 0.3 \mathrm{~km}$; zone $4: 3.5 \mathrm{~km}$.

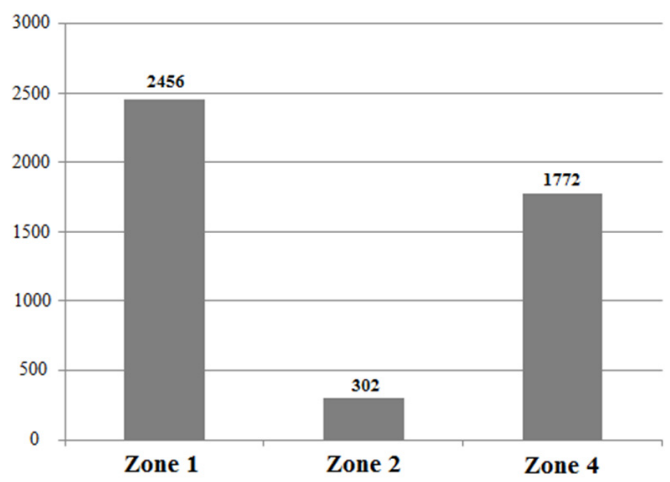

Figure 7: Fish biomass $\left(\mathrm{g} / 100 \mathrm{~m}^{2}\right)$. Comparison between zones 1, 2 and 4 .

The removal of the Aval Rabaud weir has been conducted the 24th of July 2014 at 9:00 am (first arrow on Fig. 8(b)) and ended the 25th at 11:00 am. The first phase ( 2 hours) corresponded to the depletion of the weir and the rest of times being dedicated to modelling of the reservoir above: filling of the lateral annex and channelization.

(a)

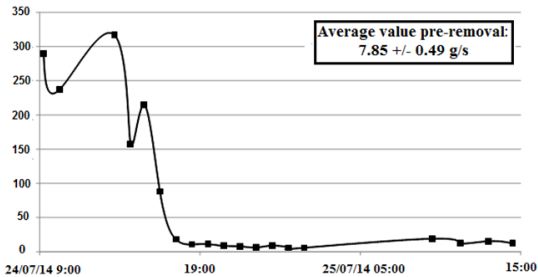

(b)

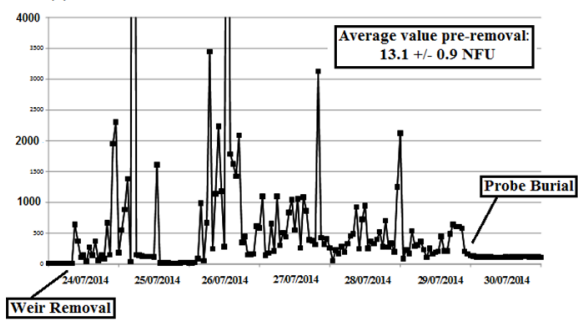

Figure 8: (a) Suspended matter (g/s) and (b) turbidity (NFU) during and after removal directly downstream of the structure. 
As expected, a strong release of fine sediments from the reservoir to the river downstream was observed (Fig. 8). Visual observations during and after the works showed a transfer of sediments far away downstream beyond the limits of the study area. This strong discharge (300 g/s) occurred immediately after the beginning of the operation and took place over a period of 9 hours (Fig. 8(a)). The behaviour of suspended matter flux observed on Fig. 8 is due to the operation of weir destruction and the work of an excavator directly in the riverbed.

Even though the suspended sediments discharge only took place during the first day of the operations, the measure of turbidity by the probe placed bottom of riverbed recorded a bed load phenomenon occurring in the days following the removal (Fig. 8(b)). Analysis of turbidity variations over the first days after operations highlighted this phenomenon throughout 6 days before the burial of the probe under sediments (second arrow in Fig. 8(b)). It is therefore likely that the extent and duration of the sediment discharge is currently underestimated mainly concerning bed load occurring a long time after work.

The types of habitat and the flow profiles observed in zones 1 and 4 have not been highly influenced by the removal of the middle weir number 2 due to the persistence of the two others. Thus only zones 2 and 3 have been prospected in post-removal campaign. Results show the short/medium term evolution (few months after work) of the study site in response to the erasing of weir 2 .

In zone 2 the strong sediment discharge observed in Fig. 9, has led to a net increase in fine substrate over the entire area (twice as great). In zone 3, transport of fines downstream has resulted in the emergence of coarser material, mainly boulders. Yet fine sediments remain the dominant substrate $(65 \%)$. The presence of a natural barrier remaining after the work (waterfall generated by bedrock) creates a reduced retention area. Moreover the persistence of weir 3 upstream blocks the charge in coarser sediment. These two reasons contribute to maintain the domination of fine substrate in zone 3.
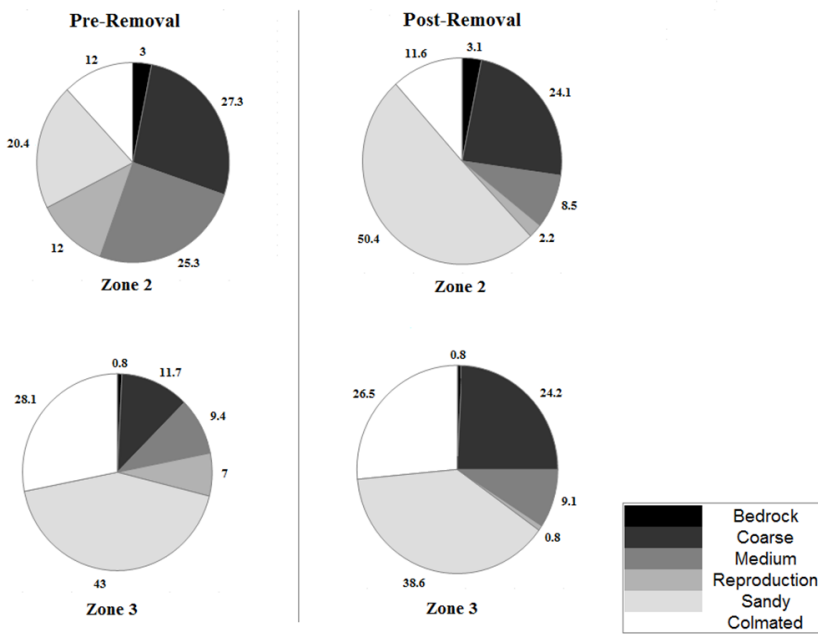

Figure 9: Habitats before and after removal in zones 2 and 3. 
Flow profiles have radically changed, showing a shift to a mostly lotic environment in comparison with the slow-flowing facies observed during preremoval campaigns. In zone 3, the restoration of the reservoir upstream of weir (filled of lateral annex, channelization and stabilization of banks), leads to a decrease in bed width. The same decrease and a channelization have also been observed upstream on a non-artificially modified linear, demonstrating the resumption of natural evolution. In zone 2, the discharge of sediment and the increase of fine substrate induce a slightly decrease of lotic profile (dominant one) but the diversity of flow is maintain. Thus elimination of weir 2 has not serious issue on flow profile downstream but improves greatly upstream one.
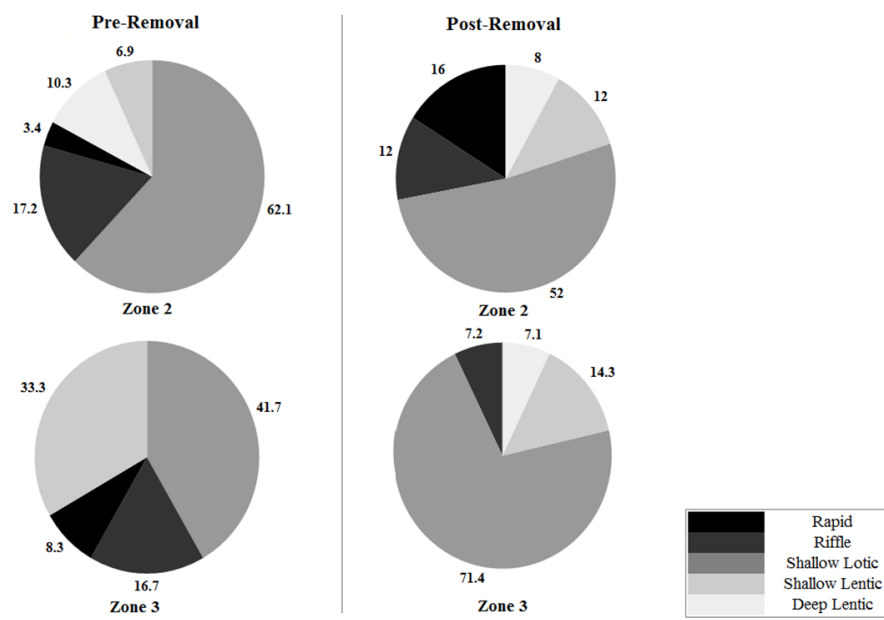

Figure 10: Flow profiles before and after removal in zones 2 and 3.

\section{Conclusion}

The pre-removal campaigns suggested a strong influence of weirs on river equilibrium (flow, habitat, fishes).

Fish inventories showed that the impact on the communities is linked to the fragmentation of ecosystem more than to the diversity of available substrates. Indeed results have shown that the sections exhibiting less biomass are the most limited one in terms of linear length. It seems therefore reasonable to say that the removal of the 2 structures inducing the re-connection of $12 \mathrm{~km}$ of river, will lead to a great improvement for fishes community in term of variety and biomass.

Morphological measures showed that every area directly above a transversal structure is largely disturbed. It has been observed a significant decrease in habitat diversity and quality from downstream to upstream. The succession of closely located obstacles induces retention of fine sediments leading to the progressive clogging of the upstream substrate.

The smoothing of the slope in upstream areas caused the dominance of lentic dynamics. 
The removal operation caused a strong release of fine sediments retained over the years upstream of the weir. This discharge has uncovered several boulders upstream, and contributed to incision of bed and channelization.

Upstream of the deleted weir a reduction in the bed width is observed. Part of this evolution is due to artificial changes made during the work. Nevertheless, beyond this area the stream presents a channelization due to re-establishment of fluvial dynamics. In this section the stream dynamics have increased, returning to a more natural behaviour.

However, a few months after removal the evolution of the stream does not show any apparition of medium sized sediments such has cobbles and gravels essential to reproduction and development of lotic fish communities. The cause here is the presence of the upstream weir blocking any sedimentary renewal. Thus, the elimination of the structure is only a step to re-naturing of site and the levelling of the near weir upstream is necessary to the success of the rehabilitation project.

The negative effect of the operation expressed by a high release of fine sediment downstream contributed to the degradation of local environment quality. Nonetheless, such input seems not sufficient to permanently damage downstream area. Moreover, it is believe that the seasonal increase in flow rates will transport these particles further downstream. The area will then return to its previous good state.

\section{References}

[1] Petts G.E., 2008. Instream flow science for sustainable river management. Journal of the American Water Resources Association 45, pp. 1071-1086, 2008.

[2] Musil, J., Horky, P., Slavík, O., Zboril, A., Horká, P., The response of the young of the year fish to river obstacles: functional and numerical linkages between dams, weirs, fish habitat guilds and biotic integrity across large spatial scale. Ecological Indicators 23, pp. 634-640, 2012.

[3] Dongkyun, I., Hyeongsik, K., Kyu-Ho, K., Sung-Uk C., Changes of river morphology and physical fish habitat following weir removal. Ecological Engineering 37, pp. 883-892, 2011.

[4] Malavoi, J.R., Ouvrages transversaux sur les cours d'eau: impacts hydromorphologiques et écologiques et principes de restauration globale. ONEMA 2009.

[5] Gangloff M.M., Taxonomic and ecological tradeoffs associated with small dam removals. Aquatic Conserv: Mar. Freshw. Ecosyst. 23, pp. 475-480, 2013.

[6] Santucci, jr V.J., Gephard, S.R., Pescitelli, S.M., Effects of multiple lowhead dams on fish, macroinvertebrates, habitat, and water quality in the Fox River, Illinois. North American Journal of Fisheries Management 25, pp. 975-992, 2005.

[7] Stanley, E.H., Doyle, M.W., Trading off: the ecological effects of dam removal. Frontiers in Ecology and the Environment 1, pp. 15-22, 2003. 
[8] Doyle, M.W., Stanley, E.H., Orr, C.H., et al., Stream ecosystem response to small dam removal: Lessons from the Heartland. Geomorphology 71, pp. 227-44, 2005.

[9] Stanley, E.H., Luebke, M.A., Doyle, M.W., Marshall, D.W., Short-term changes in channel form and macroinvertebrate communities following low-head dam removal in the Baraboo River, Wisconsin. Journal of the North American Benthological Society 21, pp. 172-187, 2002.

[10] Malavoi, J.R., Souchon, Y., Description standardisée des principaux faciès d'écoulement observables en rivière : clé de détermination qualitative et mesures physiques, Bull. Fr. Pêche Piscic. 365/366, pp. 357-372, 2002.

[11] Frissell, C.A., Liss, W.J., Warren, C.E., Hurley, M.D., A hierarchical framework for stream habitat classification: viewing streams in a watershed context, Environmental Management 10(2), pp. 199-214, 1986. 